\title{
Constrained predictive control of a counter-current extractor
}

\author{
M. Mulholland, N.K. Narotam \\ Department of Chemical Engineering, University of Natal, \\ King George V Avenue, Durban 4001, South Africa. \\ Tel: +27-31-2603123. Fax: +27-31-2601118. \\ e-mail: mulhollal che. und.ac. za
}

\begin{abstract}
Continuous counter-current liquid-liquid extraction of copper from an acidic aqueous solution is performed in a $6 \mathrm{~m}$ pulsed packed column. Bubbles of an immiscible organic phase, containing the complexing agent LIX $64 \mathrm{~N}$, move upward as the continuous aqueous phase moves downward. It is desired to regulate the residual copper content of the departing aqueous phase, using as control actions the organic feed rate and the pulse frequency. In this distributed system, profiles of copper concentration become imprinted on the descending aqueous phase as a result of the history of controls and disturbances acting during the passage of a parcel. Initial work has involved the development of a dynamic model of the extractor, and the proving of a constrained model predictive controller on this model. The model is based on a spectral solution along the discretised column length, with special treatments of convection and the boundary conditions. The Linear Dynamic Matrix Controller (LDMC) minimises the summed magnitudes of deviation from setpoint, over a time horizon of up to 50 steps. The algorithm used is based on the method of Chang and Seborg (1983).
\end{abstract}

\section{Keywords}

Model predictive control, distributed system, liquid-liquid extraction, spectral model, linear dynamic matrix control.

\section{INTRODUCTION}

Acid leach liquors containing metallic ions result from metallurgical extraction processes. One means of concentrating the metals for recovery is by extraction into an organic phase (eg. kerosene) containing a metal-ion complexing agent such as LIX 64N (Forrest and Hughes, 1974; Kordosky, et al, 1987; Mickler and Uhlemann, 1992). This may be done by contacting the two phases in a series of mixer-settlers, or, as in this case (Figure 1), in a packed tower (Kumar and Hartland, 1994). Raschig ring packing in our tower breaks up the rising organic bubbles to offer more surface area for the inter-phase transfer to take place. This effect can be enhanced by pulsing at the base to superimpose an oscillatory vertical motion on the mean flow. 

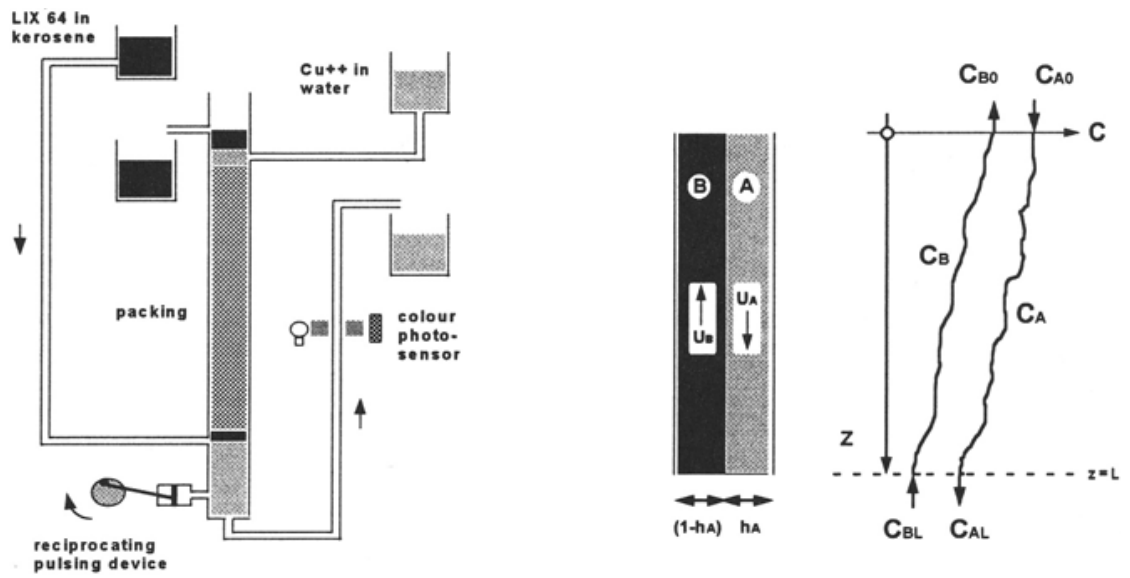

Figure 1 Arrangement of $6 \mathrm{~m}$ pulsed packed column for the extraction of $\mathrm{Cu}^{++}$from aqueous solution into kerosene containing LIX 64.

\section{MODELLING}

The system may be described by the pair of equations (Tsouris and Tavlarides, 1990; Hufnagel et al, 1991):

$$
\begin{aligned}
& \frac{\partial C_{A}}{\partial t}=-u_{A} \frac{\partial C_{A}}{\partial z}+D_{A} \frac{\partial^{2} C_{A}}{\partial z^{2}}-\frac{k a}{h_{A}}\left(C_{A}-\varepsilon C_{B}\right) \\
& \frac{\partial C_{B}}{\partial t}=+u_{B} \frac{\partial C_{B}}{\partial z}+D_{B} \frac{\partial^{2} C_{B}}{\partial z^{2}}+\frac{k a}{\left(1-h_{A}\right)}\left(C_{A}-\varepsilon C_{B}\right)
\end{aligned}
$$

where $C_{A}$ and $C_{B}$ are the concentrations of copper in the aqueous and organic phases respectively. The term $k a$ (transfer coefficient $\times$ surface area per unit volume) is expected to rise with more vigorous pulsing. Once the volumetric feeds $f_{A}$ and $f_{B}$ to the column are set, a characteristic volumetric holdup fraction is occupied by each phase, $h_{A}$ and $h_{B}=\left(1-h_{A}\right)$, depending on which phase becomes continuous, and which is dispersed. The velocities are then determined by $u_{A}=f_{A} /\left[h_{A} A\right]$ and $u_{B}=f_{B} /\left[\left(l-h_{A}\right) A\right]$.

Notice that in this representation, the equilibrium concentration in the aqueous phase $C_{A}{ }^{*}$, which would correspond to the present concentration $C_{B}$ in the organic phase, is approximated as being proportional $(\varepsilon)$ to $C_{B}$.

Various dynamic models of liquid-liquid extraction columns have been presented. Yoswathana et al (1985) used a simple lumped second-order discrete model for the aqueous exit concentration (conductivity). Bart, Bauer and Marr (1987) considered a detailed equilibrium mechanism, but only 
solved for the concentration profile in the column at equilibrium. The modelling approach of Najim et al (1987) is implicit, in that the algorithm treats the on-line process as a black box, and identifies those control levels which appear more successful, giving increasing weight to them. Najim et al (1988a, 1988b) use a lumped 3rd-order discrete model to represent the dependence of exit concentration (conductivity) on the pulse frequency. Najim (1988) reverts to the stochastic learning algorithm of Najim et al (1987), but adds a second control action, viz. the organic feed rate, to the pulse frequency, and includes a "feedforward" term in the form of a second conductivity measurement at some point in the column. Najim and Irving (1989) again used a lumped secondorder SISO model for a liquid-liquid extractor. Najim and Youlal (1990) based a real-time identification on a lumped second-order discrete model, for pole-placement adaptive control. Tsouris and Tavlarides $(1990,1991)$ stress that an increase in agitation (eg. pulsing) benefits extraction partly by increasing the organic phase holdup. They model this dependence of holdup on agitation as first order plus dead-time lag.

In the present work, it was recognised that the uniform velocity and axial diffusivity within each phase would lead to a simple solution by Fourier-transforming the dependence on $z$. Represent the two profiles of concentration by the single bivariate function

$C_{j k}=C(y, z)$ with $y=j \Delta y, j=1,2$ and $z=k \Delta z, k=1, N$

such that $C_{1 k}=C_{A}(k \Delta z)$ and $C_{2 k}=C_{B}(k \Delta z)$.

If we have the 2-dimensional discrete Fourier transform of this function, it can be expressed in terms of the Fourier coefficients $A_{j k}$ using the inverse Fourier transform:

$C(y, z)=\frac{1}{2 N} \sum_{k=1}^{N} \sum_{j=1}^{2} A_{j k} \exp \left\{-i 2 \pi\left[\frac{(j-1) y}{2 \Delta y}+\frac{(k-1) z}{2 \Delta z}\right]\right\}=\frac{1}{2 N} \sum_{k=1}^{N} \sum_{j=1}^{2} A_{j k} E_{j k}$

Defining $f_{k}=-\mathrm{i} 2 \pi k /(N \Delta z)$, note that

$$
\begin{aligned}
& \frac{\partial C}{\partial z}=\frac{1}{2 N} \sum_{k=1}^{N} \sum_{j=1}^{2} f_{k} A_{j k} E_{j k} \\
& \frac{\partial^{2} C}{\partial z^{2}}=\frac{1}{2 N} \sum_{k=1}^{N} \sum_{j=1}^{2} f_{k}^{2} A_{j k} E_{j k}
\end{aligned}
$$

Substitute (3),(4) and (5) into (1) and (2) and equate the $k$ harmonics to obtain

$$
\begin{aligned}
& \frac{\partial A_{1 k}}{\partial t}+\frac{\partial A_{2 k}}{\partial t}=\left(-u_{A} f_{k}+D_{A} f_{k}^{2}-k a / h_{A}\right)\left(A_{1 k}+A_{2 k}\right)+\left(k a \varepsilon / h_{A}\right)\left(A_{1 k}-A_{2 k}\right)=f_{A 1 k} A_{1 k}+f_{A 2 k} A_{2 k} \\
& \frac{\partial A_{1 k}}{\partial t}-\frac{\partial A_{2 k}}{\partial t}=\left(+u_{B} f_{k}+D_{B} f_{k}^{2}-k a \varepsilon / h_{B}\right)\left(A_{1 k}-A_{2 k}\right)+\left(k a / h_{B}\right)\left(A_{1 k}+A_{2 k}\right)=f_{B 1 k} A_{1 k}+f_{B 2 k} A_{2 k}
\end{aligned}
$$

Thus

$$
\frac{\partial A_{k}}{\partial t}=\boldsymbol{M}_{k} \boldsymbol{A}_{k} \quad \text { with } \quad \mathbf{A}_{k}=\left(\begin{array}{l}
A_{1 k} \\
A_{2 k}
\end{array}\right) \quad \text { and } \quad \mathbf{M}_{k}=\frac{1}{2}\left[\begin{array}{ll}
\left(f_{A 1 k}-f_{B 1 k}\right) & \left(f_{A 2 k}-f_{B 2 k}\right) \\
\left(f_{A 1 k}+f_{B 1 k}\right) & \left(f_{A 2 k}+f_{B 2 k}\right)
\end{array}\right]
$$


Integrate using the matrix exponential:

$$
A_{k}(t+\Delta t)=\exp \left(M_{k} \Delta t\right) A_{k}(t)
$$

whence $C_{A}(z, t+\Delta t)$ and $C_{B}(z, t+\Delta t)$ are found using the inverse Fourier transform (3).

Since the Fourier transform is periodic, boundary conditions are handled by including in the solution, extensions of the $z$-range, at both ends of the column, which are longer than the convective jump on each time-step $\Delta t$. On each time-step, the concentration in this region is set to the feed condition of each phase, at the appropriate feed end. At the remote end, the "external" concentration is reset to the immediate internal concentration.

The above spectral solution is not accurate for convective jumps involving fractions of $\Delta z$. A minor approximation is made to overcome this problem for each phase. Records are kept of the "true" and "solution" distances of travel of each phase:

$$
\begin{array}{ll}
d_{\text {Atrue }}=\int_{0}^{t} u_{A}\left(t^{\prime}\right) d t^{\prime} & d_{\text {Asoln }}=\int_{0}^{t} u_{\text {Aadj }}\left(t^{\prime}\right) d t^{\prime} \\
d_{\text {Btrue }}=\int_{0}^{t} u_{B}\left(t^{\prime}\right) d t^{\prime} & d_{\text {Bsoln }}=\int_{0}^{t} u_{\text {Badj }}\left(t^{\prime}\right) d t^{\prime}
\end{array}
$$

On each time-step, adjusted velocities $u_{\text {Aadj }}$ and $u_{B a d j}$ are recalculated for use in the solution, such that they cause the solution travel distance to fall on the whole number of steps $\Delta z$ which lies closest to the true travel distance for each phase.

\section{CONTROL ALGORITHM}

Several workers have considered the control problem for this distributed system. Yoswathana et al (1985) recursively estimated parameters for a lumped second-order discrete model on-line, for poleplacement adaptive control using a discrete PID controller. Najim et al (1987) and Najim (1988) used "black box" learning algorithm which automatically re-inforced controls which were detected to have a beneficial effect. Najim et al (1988a, 1988b) used an adaptive linear quadratic controller based on identified parameters for a 3rd-order discrete SISO model of the process. Najim and Irving (1989) used an unconstrained model predictive controller based on the discrete second-order lumped model relating exit conductivity to the pulse frequency. Najim and Youlal (1990) used poleplacement adaptive control with a regularization procedure to avoid the pole-zero cancellation problem.

In the present approach, it was recognised that an ideal controller should predict and counteract perturbations of the aqueous concentration profile moving towards the exit point. Both control actions will affect the entire concentration profile, so some minimisation of future deviations from setpoint over a time horizon is essential. A constrained multivariable Linear Dynamic Matrix Controller (LDMC), based on the linear programming solution of Chang and Seborg (1983), and the formulation of Morshedi et al (1985), has been formulated as follows:

Consider a vector of input control actions $m$, which might contain both the organic phase feed rate and the pulse frequency. For a linear system, the response of the output vector $x$ (eg. aqueous and organic concentrations) at the next $N$ intervals can be constructed by superposition of the step responses for the sequence of control vector moves $\Delta m_{1}, \Delta m_{2}, \ldots, \Delta m_{N}$ as follows: 


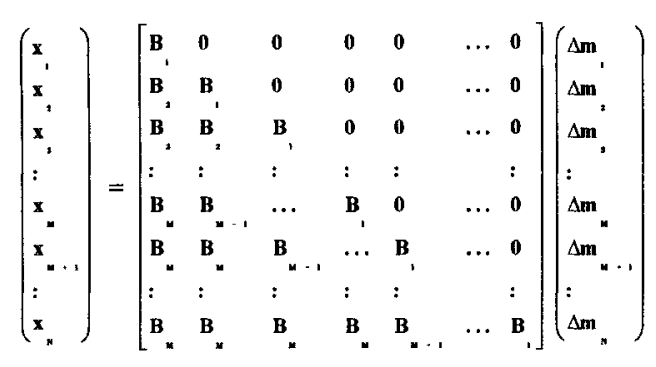

(Steady - state response achieved $M$ steps ahead with $M<N$ )

We represent this convolution model for future outputs as $\boldsymbol{x}=\boldsymbol{B} \Delta \boldsymbol{m}$. Now, defining the $N \boldsymbol{x} M$ matrices:

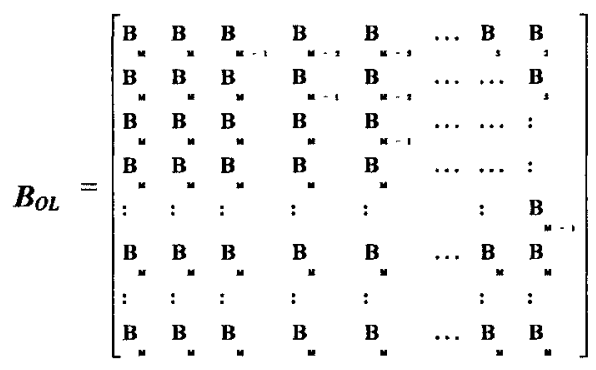

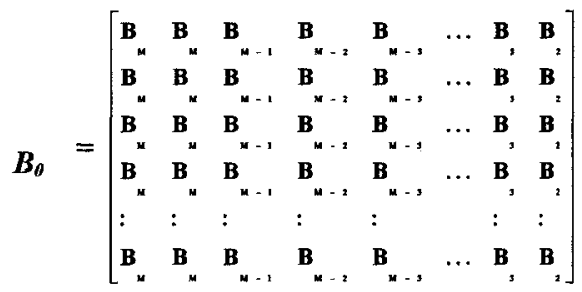

and the present measurements $(N)$ and past inputs $(M)$ :

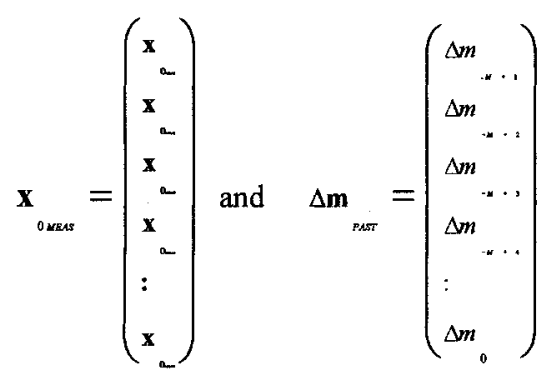


then the "open-loop" response, corrected for present model offset, is

$\boldsymbol{x}_{O L}=\boldsymbol{x}_{O M E A S}+\left[\boldsymbol{B}_{O L}-B_{\theta}\right] \Delta \boldsymbol{m}_{P A S T}$

and the "closed loop" response up to the $N$-step horizon is obtained by including the contribution of the future control input steps $\Delta m$ :

$\boldsymbol{x}_{C L}=\boldsymbol{x}_{O L}+B \Delta \boldsymbol{m}$

On each time-step it is possible to compute the future open-loop response $x_{O L}$ based on past inputs and the present output. Thus the control problem amounts to finding suitable $\Delta m$. Such an optimisation might solve for a limited sequence of the steps $\Delta m_{1}, \Delta m_{2}, \ldots, \Delta m_{N}$, but it is only the first step $\Delta m_{1}$ which is actually implemented, before the entire computation is repeated on the next timestep.

Define $x_{S P}$ to contain a sequence of set-points for the outputs up to the time horizon, so that the open-loop error may be calculated in advance as $x_{O L}-x_{S P}$. Then the ideal control sequence $\Delta m$ will be the one which minimises the magnitudes of the elements of the residual

$r=x_{O L}-x_{S P}+B \Delta m$

Transforming and adding a term for move suppression (Morshedi et al, 1985), we minimise rather the magnitude of

$\rho=B^{T}\left(x_{O L}-x_{S P}\right)+\left[B^{T} B+\lambda \mathbf{I}\right] \Delta m$

Minimisation of the size of each term in $\rho$, yet keeping $\Delta m, m$ and $x_{\mathrm{CL}}$ within defined upper and lower constraints, is achieved using the linear programming technique of Chang and Seborg (1983). This requires that $\rho$ is represented by two vectors of non-negative elements, $v i z . \rho=\rho^{+}-\rho^{-}$, and that the constraints are defined in terms of

$$
\begin{aligned}
& \Delta m=\left[B^{r} B+\lambda\right]^{-1}\left[\rho^{+}-\rho^{-}-B^{T}\left(x_{O L}-x_{S P}\right)\right]=T\left[\rho^{+}-\rho^{-}-\varepsilon_{O L}\right] \\
& \boldsymbol{m}=\left[\begin{array}{lllllll}
\mathbf{I} & 0 & 0 & 0 & 0 & \ldots & 0 \\
\mathbf{I} & \mathbf{I} & 0 & 0 & 0 & \ldots & 0 \\
\mathbf{I} & \mathbf{I} & \mathbf{I} & 0 & 0 & \ldots & 0 \\
\mathbf{I} & \mathbf{I} & \mathbf{I} & \mathbf{I} & 0 & \ldots & 0 \\
: & : & : & : & : & & : \\
\mathbf{I} & \mathbf{I} & \mathbf{I} & \mathbf{I} & \mathbf{I} & \ldots & \mathbf{I}
\end{array}\right] T\left[\rho^{+}-\rho^{-}-\varepsilon_{O L}\right] \\
& \boldsymbol{x}_{C L}=x_{O L}+B T\left[\rho^{+}-\rho^{-}-\varepsilon_{O L}\right]
\end{aligned}
$$

Minimisation of $w^{T}\left(\rho^{+}+\rho\right)$ within these constraints, where the vector $w$ contains a sequence of specified weights up to the time horizon, then achieves the desired result. 


\section{CONTROL APPLICATION ON MODEL}

A first application of the above LDMC algorithm has been in SISO control of the countercurrent extractor model. The manipulated control action is the organic phase feed rate, with the 'aqueous exit $\mathrm{Cu}^{++}$composition being the controlled process variable. The convolution model of the process was based on $M=50$ points of a step response near the normal operating point. Figure 2(a) shows the servo- and load-performance when only the first control step has been used to optimise the closed-loop response over a horizon of $N=50$ steps. Notice the characteristic behaviour of the LDMC in which the control action moves directly to the compensation level following a step-change in set-point, or a step disturbance. For comparison, a proportional-integral controller was tuned using the recommended Ziegler-Nichols settings obtained from the reaction curve. The performance with the same set-point and disturbance sequence is shown in Figure 2(b). The system non-linearity results in differing behaviour of the PI controller over the organic phase flow range. Appreciable control action cycling is necessary to hold the exit $\mathrm{Cu}^{++}$ composition at set-point. Further advantages are expected from the LDMC approach for MIMO case, in which both organic phase feed rate $\left(f_{B}\right)$ and the pulsing frequency (affecting $k a$ ) will be used as control actions.

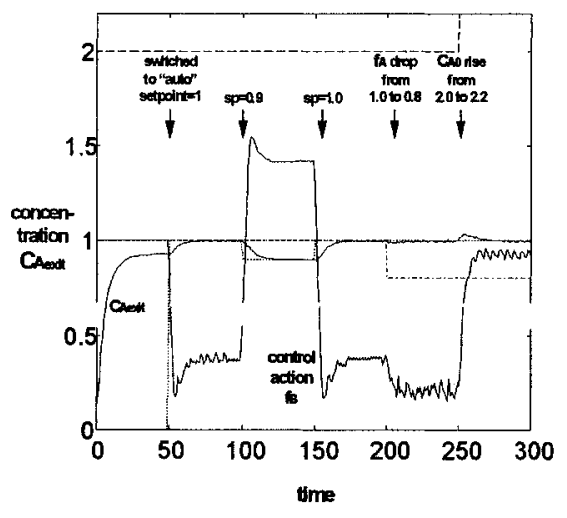

(a) Linear Dynamic Matrix Control

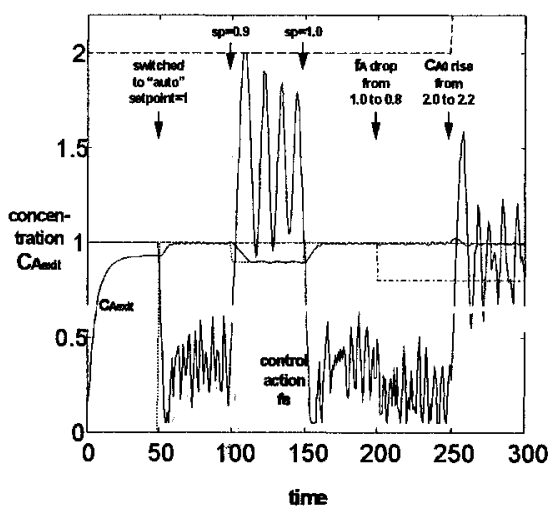

(b) Proportional-Integral Control

Figure 2 Control of exit $\mathrm{Cu}^{++}\left(C_{A \text { exit }}\right)$ by manipulation of organic phase feed rate $f_{B}$.

\section{ACKNOWLEDGEMENTS}

The support of the Foundation for Research Development and the University of Natal Research Fund is gratefully acknowledged. 


\section{REFERENCES}

Bart H.J., Bauer A. and Marr R. (1987) Modelling of mass transfer with chemical reaction in solvent extraction equipment, Technische Universitat Graz, Austria.

Chang T.S. and Seborg D.E. (1983) A linear programming approach for multivariable feedback control with inequality constraints, Int.J.Control 37, 3, 583-597.

Forrest C. and Hughes M.A. (1974) The modelling of equilibrium data for the liquid-liquid extraction of metals: Part II: Models for the copper/LIX64 and chromate/ALIQUAT336 systems, Hydrometallurgy I, 139-154.

Hufnagel H., McIntyre M. and Bla $\beta$ E. (1991) Dynamic behaviour and simulation of a liquid-liquid extraction column, Chem Eng. Technol 14, 301-306.

Kordosky G.A., Olafson S.M., Lewis R.G. and Deffner V.L. (1987) A state-of-the-art discussion on the solvent extraction reagents used for the recovery of copper from dilute sulfuric acid leach solutions, Separation Science \& Technology 22(2\&3), 215-232.

Kumar A. and Hartland S. (1994) Prediction of drop size, dispersed-phase holdup, slip velocity, and limiting throughputs in packed extraction columns, Trans IChemE 72A, 89-104.

Mickler W. and Uhlemann E. (1992) Liquid-liquid extraction of copper from ammoniacal solution with $\beta$-diketones, Separation Science and Technology 27(12), 1669-1674.

Morshedi A.M., Cutler C.R. and Skrovanek T.A. (1985) Optimal solution of Dynamic Matrix Control with Linear Programming techniques (LDMC), American Control Conference, Boston.

Najim K., Le Lann M.U. and Casamatta G. (1987) Learning control of a pulsed liquid-liquid extraction column, Chemical Engineering Science 42, 7, 1619-1628.

Najim K., Irving E., Youlal H. and Najim M. (1988) Adaptive linear quadratic control of an extractor, IFAC Symposium on Adaptive Control of Chemical Processes, Copenhagen.

Najim K., Youlal H., Irving E. and Najim M. (1988) Linear quadratic self-tuning control of a liquidliquid extraction column, Optimal Control Applications \& Methods 9, 273-284.

Najim K. (1988) Multivariable control of a liquid-liquid extraction column using a probabilistic automaton, IEE Proceedings 135D, 6.

Najim K. and Irving E. (1989) Extended horizon self-tuning control of a pulsed liquid-liquid extraction column, Optimal Control Applications and Methods 10, 375-383.

Najim K. and Youlal H. (1990) Regularized pole-placement adaptive control of a liquid-liquid extraction column, Int.J.Systems Sci 21, 7, 1313-1323.

Tsouris C. and Tavlarides L.L. (1990) Application of the ultrasonic technique for real-time holdup monitoring and control of extraction columns, Chemical Engineering Science 45, 10, 30553062.

Tsouris C. and Tavlarides L.L. (1991) Control of dispersed-phase volume fraction in multistage extraction columns, Chemical Engineering Science 46, 11, 2857-2865.

Yoswathana N., Casamatta G. and Najim K. (1985) Adaptive control of a liquid-liquid extractor, IFAC Symposium on Adaptive Control of Chemical Processes, Frankfurt. 